EPSC Abstracts

Vol. 14, EPSC2020-876, 2020, updated on 21 Jan 2021

https://doi.org/10.5194/epsc2020-876

Europlanet Science Congress 2020

(C) Author(s) 2021. This work is distributed under

the Creative Commons Attribution 4.0 License.

\title{
Experimental scattering matrix of olivine: the effect of the particles size
}

Elisa Frattin ${ }^{1,3}$, Teresa Jardiel ${ }^{2}$, Olga Muñoz ${ }^{3}$, Juan-Carlos Gomez-Martin ${ }^{3}$, Fernando Moreno ${ }^{3}$, and Amador Caballero ${ }^{2}$

${ }^{1}$ University of Padua, Department of Physics and Astronomy, Vicolo dell'Osservatorio 3, 35122 Padova, Italy

(elisa.frattin@gmail.com)

${ }^{2}$ Instituto de Cerámica y Vidrio, CSIC, C/Kelsen 5, Campus Cantoblanco, E-28049 Madrid, Spain

${ }^{3}$ Instituto de Astrofísica de Andalucía, CSIC, Granada 18008, Spain

\section{Abstract}

We present the experimental curves of phase function and linear polarization degree of five samples of olivine with di different size distributions.

These unique measurements allow for the first time to disentangle experimentally the effect of the particle size on the scattering matrix

elements. The high level of accuracy is given by the very narrow and monomodal size distributions that allow individuating the interval of size

responsible for a peculiar feature of the curves.

\section{Introduction}

Dust is a primary constituent of planetary systems and a key element to study the physical and chemical conditions involved in the formation and evolution of planetesimals in the protoplanetary disk. The investigation of the scattering of light by particles of various properties in a controlled environment, such as the laboratory, is of fundamental importance to interpret the observational data. The measurement presented in this work has been taken at the IAA Cosmic Dust Laboratory (CODULAB) [4]. The facility is devoted to the measurement of the scattering matrix of a randomly oriented cloud of particles. The angular distribution of the scattered intensity by the dust and their degree of linear polarization are intimately related to the nature of those particles. Since each material has its own characteristic features, the scattering theory can be used to interpret observations

and retrieve information about the dust properties. Therefore, the laboratory data are used as a reference for comparison and interpretation,

allowing a correct analysis of the observational data. The measurements performed in this work clearly illustrate the effect of the size of the particles on the scattering matrix elements. 


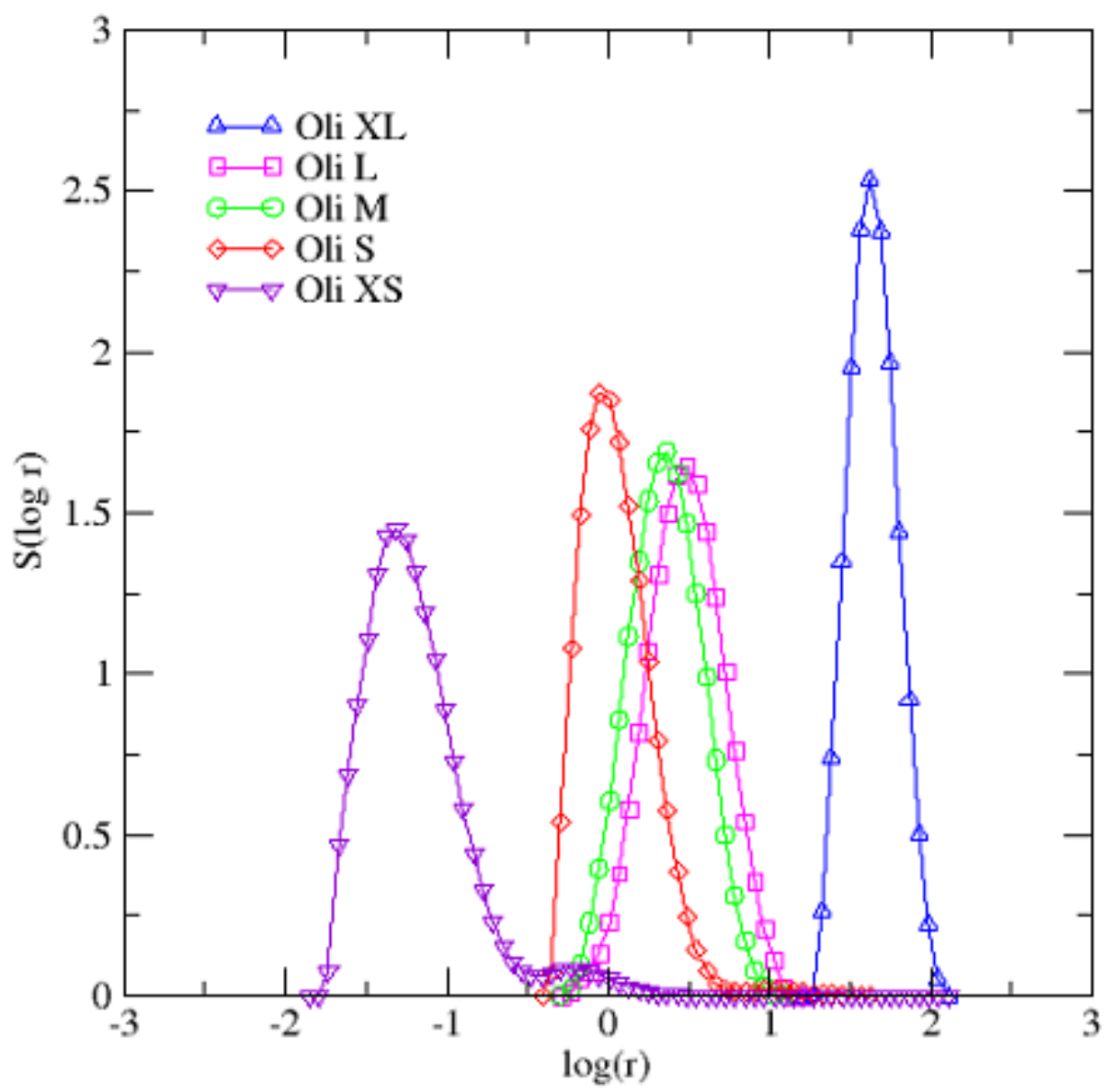

Figure 1. Size distributions

\section{Sample characterization}

The material selected for the samples is magnesium-rich olivine with a 5-10 \% of iron. The two principal phases detected by $\mathrm{X}$-rays diffraction are $(\mathrm{Mg}, \mathrm{Fe})_{2} \mathrm{SiO}_{4}$ and $\mathrm{Ca}(\mathrm{Al}, \mathrm{Si}) \mathrm{O}_{\mathrm{x}}$. Olivine is a material that has been found in comets, asteroids and IDPs [1]. The particulate of all the samples is characterized by irregular shapes and sharp edges. The decantation and centrifugation processes, using wet media assure to clean the particles from smaller components, leading to a very smooth surface. Figure 1 shows the size distributions of the 5 samples. All the size distributions of the samples are very narrow and monomodal, therefore they can be characterized by the effective radius $r_{\text {eff }}$, the effective variance $\sigma_{\text {eff }}$ and the size parameter computed from the effective radius $x_{\text {eff }}=2 \pi r_{\text {eff }} / \lambda$ (see Table). The scattering properties of the particles can be described according to their

belonging to one of the following three regimes: Rayleigh $\left(x_{\text {eff }}<<1\right)$, resonance $\left(x_{\text {eff }}=1\right)$ and geometric optics $\left(x_{\text {eff }}>>1\right)$. In this work we have examples of the three regimes

$\begin{array}{llll}\text { Sample } & \text { reff } & \sigma_{\text {eff }} & \text { xeff } \\ \text { Oli XL } & 47 & 0.35 & 575 \\ \text { Oli L } & 3.5 & 0.54 & 43 \\ \text { Oli M } & 2.6 & 0.74 & 32 \\ \text { Oli S } & 1.4 & 1.0 & 17 \\ \text { Oli XS } & 0.11 & 0.38 & 1.3\end{array}$

Measurements 
We measured the phase function and the degree of linear polarization of the 5 samples. The measurements were performed at wavelength $\lambda=514 \mathrm{~nm}$, at phase angle ranging between $\mathrm{a}=3^{\circ}$ and $a=175^{\circ}$. This is the first time in which it is possible to measure the effect of the size variation of the samples on the scattering matrix elements with such an accurate level of precision. Figure 2 shows the phase functions F11 of all the samples, normalised at $a=90^{\circ}$. Overall, the curves have a rather plane trend and a strong increase in the forward direction. We observe that, as long as the size of the particles increases, the curves become flatter. Furthermore, we observe a degree of backscattering enhancement, which increases when the particle size decreases, resulting more evident for the small samples. Figure 3 shows the curves of the linear polarization. They have the characteristic bell shape for irregular particles with a negative branch at small phase angles and a peak at side angles. The main effects of the size on the linear polarization curves are the variation of the maximum of polarization and the appearance of a negative branch at small phase angles. The Pmax increases with the particle size moving from the resonance regimes to the Rayleigh domain. The best-defined and clear negative branch belongs to the sample Oli $S$, which is composed by particles of size around 1 micron, and reaches the deepest value of Pmin. Therefore, it seems that the negative branch of polarization arises in correspondence to the presence of micrometer particles. The effect of micrometer particles has also been investigated by J. Escobar-Cerezo et al. (2018) [2], who found that removing the micrometer size particles from the sample the negative branch disappeared. Other experimental measurements of micrometer particles of different composition, such as clay, meteorites and olivine show the presence of an evident negative branch $[3,4,5]$.

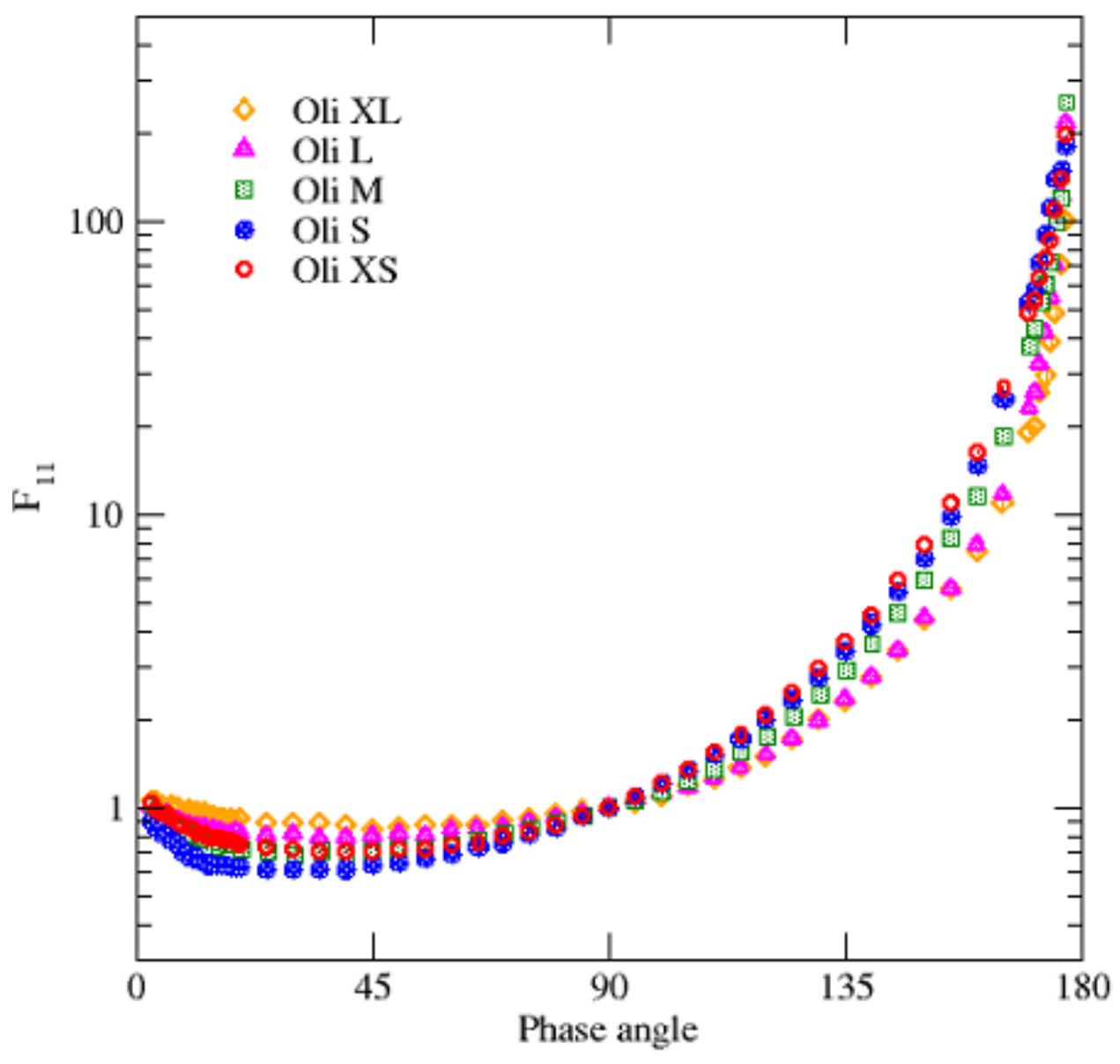

Figure 2. Phase function of the five samples 


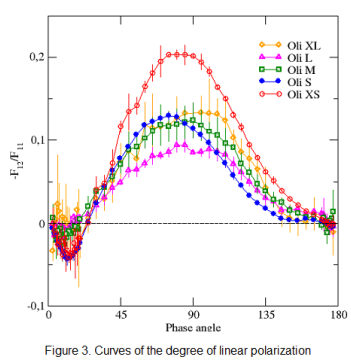

\section{References}

[1] P. Ehrenfreund et al., 2004.

[2] J. Escobar-Cerezo et al., 2018.

[3] E. Frattin et al., 2019.

[4] O. Muñoz et al., 2010.

[5] O. Muñoz et al., 2011. 